\title{
Fascitis necrosante por Apophysomyces elegans, moho de la familia mucoraceae, en paciente inmunocompetente
}

\author{
Carmen Elena Ruiz ${ }^{1}$, Myrtha Arango ${ }^{2,3}$, Ana Lucía Correa ${ }^{4}$, Luz Saider López 5 , Angela Restrepo ${ }^{2}$ \\ ${ }^{1}$ Servicio de Cirugía, Hospital Pablo Tobón Uribe, Medellín, Colombia. \\ ${ }^{2}$ Grupo de Micología Médica y Experimental, Corporación para Investigaciones Biológicas, Medellín, \\ Colombia. \\ ${ }^{3}$ Sección de Micología, Facultad de Medicina, Universidad de Antioquia, Medellín, Colombia. \\ ${ }^{4}$ Servicio de Medicina Interna, Hospital Pablo Tobón Uribe, Medellín, Colombia. \\ ${ }^{5}$ Cirugía plástica, Facultad de Medicina, Universidad de Antioquia, Medellín, Colombia.
}

Se presenta la historia de un niño de 7 años de edad, politraumatizado por accidente automovilístico, que falleció a consecuencia de infección por Apophysomyces elegans, un hongo de la familia Mucoracea. La invasión fue progresiva, inicialmente se observó una lesión puntiforme en la región lumbar izquierda que progresó a fascitis necrosante y, posteriormente, afectó la zona lumbar, los glúteos y el flanco derecho. El tratamiento antimicótico resultó inefectivo y el paciente falleció 8 semanas después de su accidente. Se presenta, además, una revisión de los casos atribuidos a este hongo.

Palabras clave: Mucormicosis (zigomicosis), trauma, fascitis necrosante, Mucoracea, Apophysomyces elegans.

\begin{abstract}
Necrotizing fasciitis in an immunocompetent patient caused by Apophysomyces elegans
A case study is presented of a 7-year-old boy, seriously injured in a car accident, who developed a fatal infection due to Aphophysomyces elegans - a mold of the Mucoracea family. Fungal invasion was initially manifested by a spotted wound in the left lumbar region which developed into a necrotizing fasciitis. Later this progressed to the right lumbar area, including the gluteus and the corresponding flank. Antimycotic treatment proved ineffective, and the child died 8 weeks after the accident. Other cases due to this fungus are reviewed.
\end{abstract}

Key words: Mucormycosis (zygomycosis), trauma, necrotizing fasciitis, Mucoracea, Apophysomyces elegans.

El término mucormicosis (zigomicosis) comprende un conjunto de entidades clínicas causadas por diversos mohos pertenecientes al orden Mucorales. Estos microorganismos se caracterizan por poseer hifas anchas, desprovistas de septos, y por ser de crecimiento rápido. Se les encuentra ampliamente distribuidos en suelos, materia orgánica en descomposición y están presentes en el aire; por consiguiente, es

Correspondencia:

Angela Restrepo, Corporación para Investigaciones Biológicas (CIB), Carrera 72 A \# 78B-141, Medellín, Colombia. Teléfono: (4) 441 0855; fax: (4) 4415514 angelares@geo.net.co

Recibido: 09/07/04; aceptado: 12/07/04 fácil entrar en contacto con sus esporas (1-4). A pesar de tan amplia distribución, la mucormicosis es relativamente infrecuente y se presenta especialmente en pacientes inmunosuprimidos con neutropenia o cáncer, en diabéticos y en desnutridos $(1,2)$. Suele diagnosticarse, igualmente, en personas inmunocompetentes que han sufrido traumas (5-10). Las infecciones se adquieren por aerosol y, también, por ingestión o inoculación traumática $(1,2,5-7,10)$.

Posiblemente, esta entidad es la afección micótica más aguda, progresiva y grave que se conoce (1$3,11-14)$. En gran parte, debido a que todos los agentes etiológicos exhiben acentuado tropismo por los vasos sanguíneos que les permite alcanzar 
diversos órganos para producir, posteriormente, émbolos y causar necrosis de los tejidos circundantes $(1-3,5,6)$.

La mucormicosis reviste diferentes formas según su localización: rinocerebral, pulmonar, cutánea, gastrointestinal y diseminada; esta última puede comprometer hueso, riñón, corazón, mediastino y otros sitios del organismo. Más de la mitad de los casos corresponde a la forma rinocerebral $(1-4,6,11)$, mientras que la cutánea representa apenas una décima parte de ellos $(1,2,5,6,8,9,12)$. Debe anotarse que el diagnóstico clínico de la mucormicosis es difícil dado que sus síntomas son similares a los de otras enfermedades $y$, también, porque inicialmente puede parecer benigno. Por consiguiente, el diagnóstico suele ser tardío por lo que la enfermedad progresa hasta volverse incontrolable (1,2,13-15). Las series de casos informados en Estados Unidos señalan que la fascitis necrosante se presenta con una frecuencia de 500 a 1.500 casos por año y que su mortalidad global está cercana al 20\% (16).

El tratamiento de la mucormicosis presenta especiales dificultades no sólo por su diagnóstico tardío sino también porque sólo se dispone de un fármaco - no siempre efectivo -, la anfotericina $B$, disponible en formulaciones a base de deoxicolato o liposomales $(1,2,14,15,17)$. Recientemente, un nuevo triazol, el posaconazol, ha mostrado ser efectivo con actividad in vitro $y$, actualmente, en etapa de ensayo clínico $(18,19)$. En casos abordables quirúrgicamente, es necesario, además, recurrir al desbridamiento de los tejidos necróticos $(1,2,9,10,12,13)$.

Se describe a continuación el caso de un niño politraumatizado quien, como consecuencia de un accidente de tránsito, desarrolló múltiples lesiones de los tejidos blandos. La inoculación traumática de un hongo de la familia Mucoraceae, Apophysomyeces elegans, resultó en infección diseminada, progresiva y fatal.

\section{Presentación del caso}

En el hospital de Corozal (Sucre) se atendió un niño de 7 años de edad, estudiante, natural y residente en Medellín, previamente sano, quien doce horas antes había sufrido politraumatismo en accidente de tránsito. El paciente presentaba distensión abdominal, taquicardia, escoriaciones y heridas en ambas rodillas, antebrazo izquierdo y cuero cabelludo; además, tenía fractura abierta de tibia izquierda. Se practicó laparotomía exploratoria, la cual no reveló lesión interna, se redujo e inmovilizó la fractura, se suturaron todas las heridas en piel y se aplicó penicilina cristalina. Por petición de la familia, se remitió a Medellín.

En urgencias del Hospital Pablo Tobón Uribe, ingresó un niño conciente, orientado en los tres planos y con sobrepeso; con frecuencia cardiaca de 100 latidos por minuto, frecuencia respiratoria de 24 por minuto, presión arterial de 129/44 mm $\mathrm{Hg}$ y presión arterial media de $72 \mathrm{~mm} \mathrm{Hg}$. Presentaba cicatriz de laparotomía superior y múltiples heridas superficiales en tórax posterior, miembros superiores e inferiores, escoriaciones y flictenas en región lumbar izquierda que llegaban hasta la parte lateral y anterior del hemiabdomen ipsilateral. Entre los exámenes de laboratorio ordenados se anotaron los siguientes: hemoglobina, 11,2 mg/dl; hematocrito, 34, y el citoquímico de orina fue normal. Se inició tratamiento con cefalotina, dipirona y líquidos endovenosos.

Tres días más tarde se halló un paciente álgido, pálido pero afebril; con frecuencia cardiaca de 108 latidos por minuto; frecuencia respiratoria de 22 por minuto; presión arterial de 124/81 mm Hg; creatinina de $0,55 \mathrm{mg} / \mathrm{dl}$; nitrógeno ureico en sangre de 15. Al ser llevado a cirugía se anotaron pequeñas áreas con quemaduras de tercer grado en glúteo izquierdo y de segundo grado en otras áreas, con flictenas y laceración en ambos flancos. En el área lumbar izquierda había una herida de $4 \mathrm{~cm} \times 0,3 \mathrm{~cm}$, aproximadamente, que comprometía piel y tejido subcutáneo y otra área de $0,3 \mathrm{~cm} \times 0,3 \mathrm{~cm}$, aproximadamente, que mostraba a su alrededor pigmentación negruzca. Además, existían varias heridas no suturadas en antebrazo izquierdo, quemadura de $1 \mathrm{~cm} \times 1 \mathrm{~cm}$ en maléolo derecho y varias laceraciones en el frontal izquierdo, incluso, una herida suturada frontoparietal de $4 \mathrm{~cm}$. En este momento se realizó lavado y desbridamiento exhaustivo, escarotomía, dermabrasión de la cara y afrontamiento de heridas. Se aplicó sulfaplata más xilocaína al 1\%, sin epinefrina. 
Se practicaron cultivos de las lesiones que, posteriormente, demostraron Pseudomonas aeruginosa (más de 100.000 UFC), Enterococcus faecalis y Staphylococcus aureus. En consecuencia, se añadió gentamicina, 160 mg por día, intravenosos, al esquema antibiótico; posteriormente, los datos del antibiograma hicieron suspender la cefalotina y la gentamicina, y se reemplazaron por ciprofloxacina y ampicilina. Al día siguiente, el paciente tenía dolor en glúteo izquierdo, genitales y presentaba, además, hidrocele postraumático doloroso. El niño se encontraba estable hemodinámicamente.

Una nueva curación en miembro inferior izquierdo hecha al séptimo día de hospitalización mostró una herida limpia en la rodilla. No se halló deterioro de otras heridas, como tampoco del estado general del paciente. Sin embargo y a pesar de la analgesia, persistía el dolor en el flanco izquierdo. Tres días más tarde, se observó celulitis en flanco izquierdo, escara café oscura en área glútea y lumbar, y también se apreció una solución de continuidad en la piel de $1,5 \mathrm{~cm}$, aproximadamente, que mostraba leve secreción purulenta y fascitis necrosante al ser desbridada. Llamó la atención que la grasa estaba condensada, era clara y densa pero en ciertas áreas tenía un color amarillo pálido (casi blanco), a diferencia de otras fascitis donde la necrosis es de carácter purulento con evidente licuefacción de la grasa $(16,20)$. Se realizó nuevo desbridamiento, escarotomía y escarectomía en glúteo. Se tomaron muestras para cultivo y se aplicó sulfaplata. Al sospechar la presencia de anaerobios se añadió metronidazol endovenoso, completando así una terapia triconjugada. Se programaron nuevos desbridamientos cada 48 horas, con cultivos cuantitativos cada vez.

Poco después de la primera semana y media de la permanencia hospitalaria se detectó necrosis grasa, la que ahora comprometía un área de 15 $\mathrm{cm} \times 18 \mathrm{~cm}$ en región lumbar y de $12 \mathrm{~cm} \times 12 \mathrm{~cm}$ en glúteo izquierdo. El paciente continuaba normotenso y estable pero taquicárdico. Había leucocitosis, neutrofilia y trombocitosis. La eritrosedimentación era de 66 y la proteína C reactiva de 17,6 mg/dl. La evaluación del infectólogo llevó a ajustar el esquema de antibióticos, continuó con ciprofloxacina y se inició ampicilina-sulbactam y gentamicina. El niño presentó febrícula y los reactantes de fase aguda iban en aumento.

A las dos semanas, al reducir en forma cerrada la fractura de tibia y colocar yeso muslopédico, se apreció que las heridas de la rodilla izquierda estaban sanas. En el tronco continuaba la necrosis fascio-grasa pero sin secreción purulenta. A las cuatro semanas, tal necrosis había afectado la región lumbar, el glúteo y el flanco izquierdo con compromiso del tejido subcutáneo y la fascia, respetando los músculos (figura 1). En este momento, el paciente entró en estado crítico y fue llevado a la unidad de cuidados especiales, donde le suspendieron la ciprofloxacina y la ampicilina sulbactam, iniciaron imipenen y continuaron con gentamicina. Para esta fecha, el paciente presentaba severa hipoproteinemia (proteínas totales, 4,3 mg/dl), con albúmina de1,0 $\mathrm{mg} / \mathrm{dl}$, había leucocitosis (52.100) y plaquetas de 567.000. Los hemocultivos para bacterias y hongos habían sido negativos.

La celulitis y la necrosis perilesional aumentaron a la semana siguiente; la lesión alcanzó la lesión un tamaño de $30 \mathrm{~cm} \times 30 \mathrm{~cm}$, aproximadamente. La necrosis se extendió a las regiones paraumbilical y paralumbar y al glúteo izquierdo. En este momento se aisló Acinetobacter baumani por lo que se añadió clindamicina para el cuadro de sepsis.

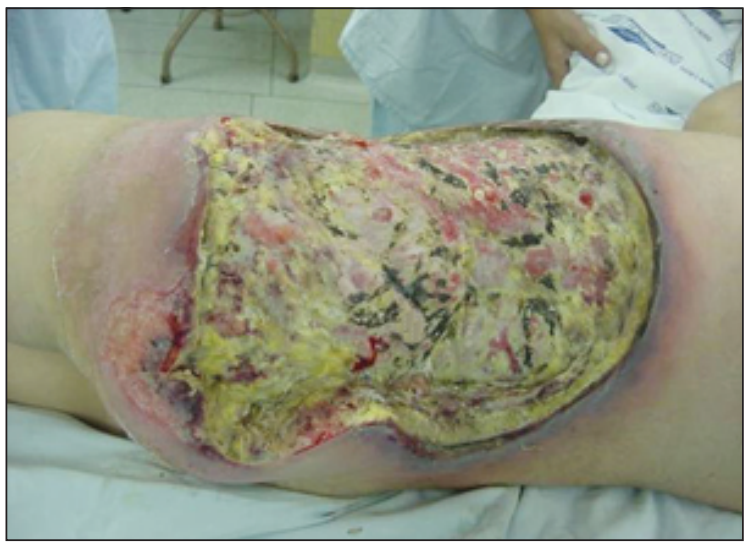

Figura 1. Paciente infectado por $A$. elegans a las cuatro semanas del trauma. En el flanco izquierdo se observa necrosis de piel, tejido subcutáneo y fascia sin compromiso del músculo. 

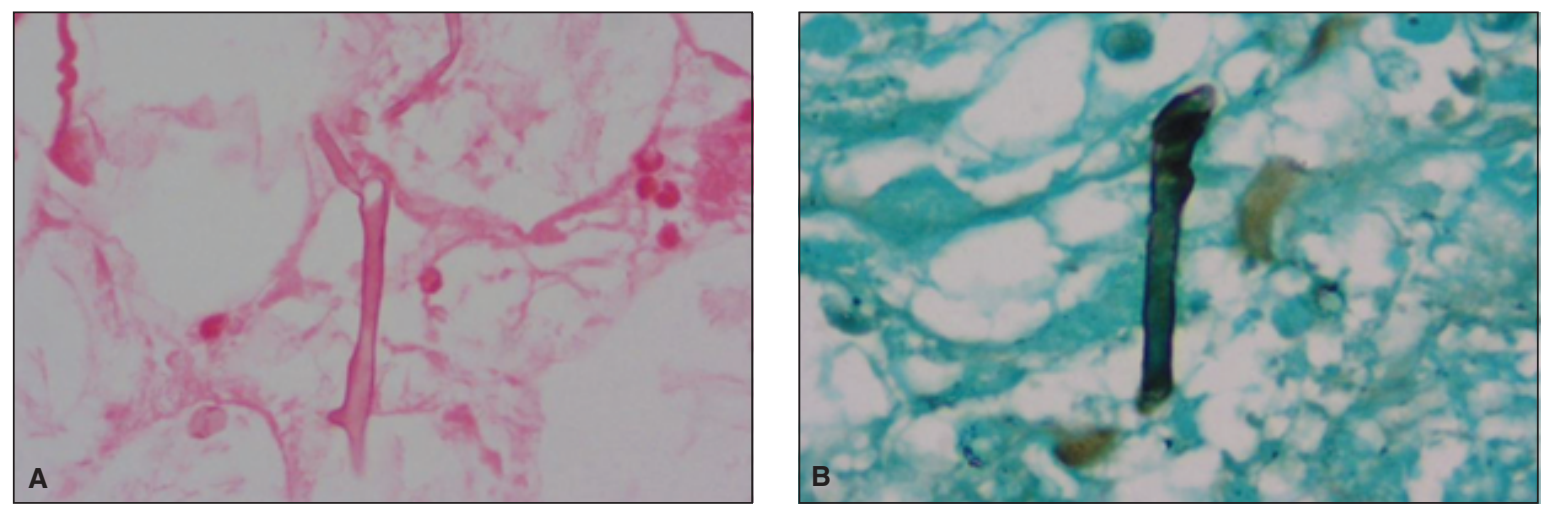

Figura 2. Biopsia de tejido necrótico: hifas aseptadas compatibles con un moho de la famillia Mucoracea. A. Hematoxilina eosina, 40X; B. Plata metenamina, 40X.

Al iniciar la cuarta semana postrauma, la fascitis necrosante ya había alcanzado la línea media del abdomen y comprometía el glúteo izquierdo, el muslo y la espalda. Mientras se realizaba el desbridamiento se presentó inestabilidad hemodinámica por lo que se suspendió el procedimiento, no sin antes tomar biopsia y muestras para nuevo cultivo. Este último reportó Staphylococcus epidermidis sensible a vancomicina. En este momento, la biopsia reportó la presencia de hifas de un hongo aseptado, compatible con Mucorales (figura 2) y se inició inmediatamente anfotericina B (20 mg por día), con reajuste de la dosis ( $30 \mathrm{mg}$ por día) 24 horas más tarde. Se continuó el tratamiento de la infección bacteriana con vancomicina, imipenen y metronidazol, además de albúmina.

Al iniciarse la quinta semana postrauma, la necrosis era casi circunferencial en abdomen, espalda izquierda, línea vertebral, con exposición del conducto deferente y del anillo inguinal (figura 3). Se aumentó la dosis de anfotericina a $40 \mathrm{mg}$ por día, atendiendo a la corrección de la hiponatremia y la hipopotasemia. El agente causal se clasificó como $A$. elegans, un agente poco común, perteneciente a la familia Mucoraceae (figura 4).

La falta de respuesta a la anfotericina después de 8 días de tratamiento (dosis acumulada de 210 $\mathrm{mg}$ ), así como su toxicidad representada por escalofríos y fiebre intensa durante la infusión, llevaron a la suspensión de la terapia. Como tratamiento de misericordia se recurrió al empleo de un nuevo antimicótico, el posaconazol, el cual había sido empleado con éxito para el tratamiento de un paciente con mucormicosis gravemente enfermo (19). Este medicamento se usó a razón de $200 \mathrm{mg}$, por vía oral, cada 8 horas por un período de 4 días.

Debido al edema del pie se retiró el yeso que cubría la fractura tibial y se encontró necrosis grasa en cara lateral, posterior y medial de la pierna, desde la región poplítea hasta el área supramaleolar. Había necrosis de vena safena a nivel inguinal y poplíteo, así como necrosis del esfínter externo por lo que no existía área anatómica óptima para una colostomía. Se hizo aparente que la mucormicosis avanzaba día a día y que los cultivos continuaban positivos, sin que los antimicóticos lograran controlar su diseminación.

Cuatro días después de iniciado el posaconazol, se reactivó la celulitis en abdomen, muslo y pierna izquierda. Había necrosis debajo del músculo glúteo con posible compromiso del nervio ciático y exposición del contenido del pliegue poplíteo. La falta de respuesta al triazol hizo necesario retornar a la anfotericina $B$ que se administró a dosis de $35 \mathrm{mg}$ por día. Sin embargo, este cambio tampoco produjo resultados ya que a la sexta semana, 14 días después de iniciado el tratamiento con antimicóticos, se apreció crecimiento abundante de un moho blanco sobre el área de necrosis de la pierna. Había, además, extenso linfedema en pie izquierdo; ninguno de los músculos expuestos revelaba la presencia de tejido de granulación. 


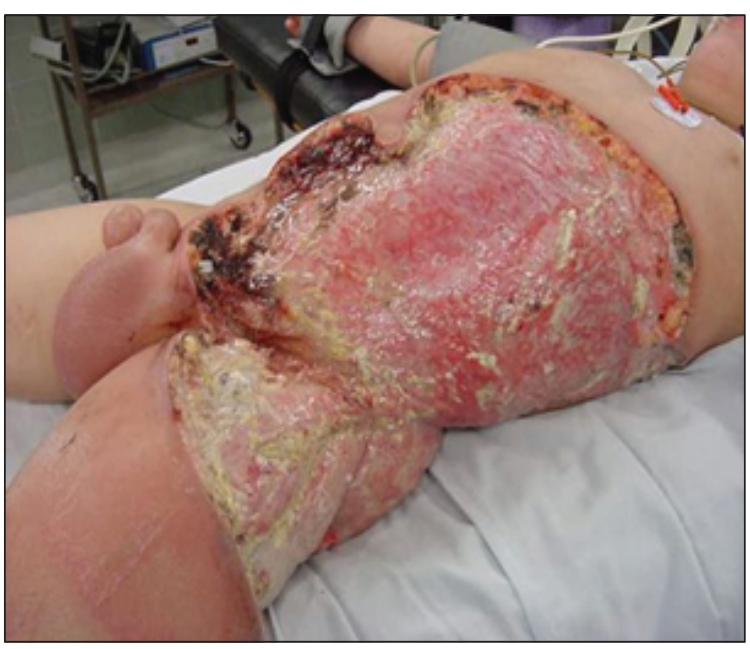

Figura 3. Quinta semana postrauma, fascitis necrosante en línea media del abdomen con compromiso del glúteo izquierdo, el muslo y la espalda. Se observa también edema testicular.

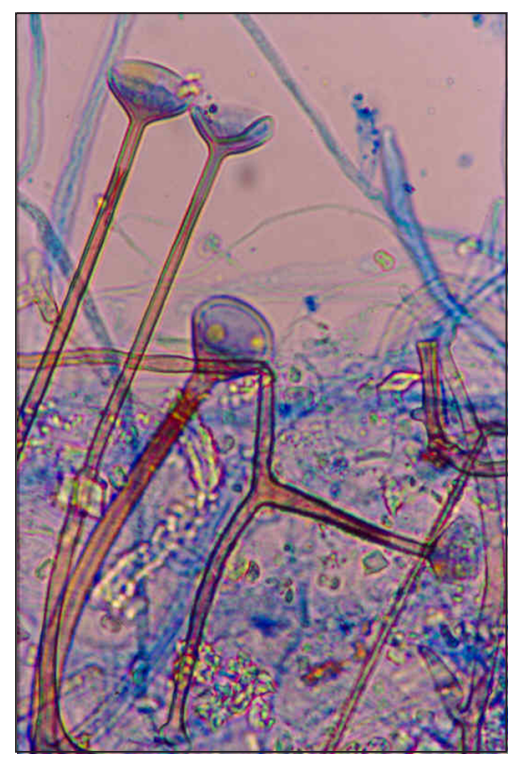

Figura 4. Apophysomyces elegans, nótese la presencia de apófisis en forma de embudo o campana. Azul de lactofenol, $60 X$.

En vista del anterior deterioro del paciente, se convocó a una reunión del grupo hospitalario encargado de la toma de decisiones en casos difíciles, el que incluyó, además, otro cirujano plástico experto en reconstrucción pediátrica. Luego de un análisis científico, ético y humano del proceso de la evolución de la enfermedad, se consideró que a pesar del tratamiento el daño tisular era del $50 \%$ y que, por tanto, existían grandes dificultades para la rehabilitación no sólo por la extensión de la lesión sino también por las áreas funcionales comprometidas. Los padres del niño fueron consultados al respecto solicitando su consentimiento para suspender el tratamiento en vista del deterioro progresivo de su hijo; ellos aceptaron esta conducta. Se decidió continuar con manejo paliativo (lavados de limpieza y analgesia), procurando el bienestar físico y mental, tanto para el niño como para su familia. El paciente falleció dos semanas más tarde, a las ocho semanas de su accidente.

\section{Discusión}

Los Mucorales son mohos ambientales saprófitos que, bajo ciertas circunstancias, pueden abandonar tal condición para causar enfermedad invasora $(1,2,6,14,19)$, como sucede en pacientes cuya resistencia está disminuida, como son aquéllos con neutropenia, diabetes mellitus, leucemia y linfoma e, igualmente, en personas previamente sanas que sufren traumas. La inoculación puede hacerse por tres rutas, respiratoria, gastrointestinal y cutánea; en esta última se incluye la infección por trauma, la aplicación de vendajes contaminados, la inyección intramuscular y otros procedimientos médicos $(1,2,6,11,14,21,22)$.

Desde el punto de vista clínico, la mucormicosis (zigomicosis) es una enfermedad invasora y grave, sin importar su agente etiológico ni su forma de presentación. La tendencia acentuada de los mucorales de invadir los vasos sanguíneos explica la rápida diseminación $(2,6,7,14,21,22)$. La mucormicosis cutánea involucra principalmente epidermis y dermis y desarrolla necrosis secundaria a la invasión micótica $(21,22)$. Las lesiones características se aprecian clínicamente 6 a 15 días postrauma. En 1987, Johnson y colaboradores intentaron clasificar la mucormicosis cutánea primaria así (9): úlceras superficiales en pacientes con diabetes mellitus; iatrogenia en paciente inmunocomprometido; celulitis gangrenosa en pacientes con trauma (fascitis necrosante); infección por adhesivos elásticos o por contaminación de quemaduras. 
Estos autores anotaron que la mucormicosis en personas previamente sanas era más benigna que en pacientes inmunosuprimidos; por ello, la profundidad, la extensión de la infección y su avance variaban mucho entre los pacientes $(6,9,10,12)$.

En general, las formas cutáneas son de dos tipos, benigna y necrosante. La primera puede ser leve y se presenta como una vesícula o pústula que tiende a ulcerarse y que, a veces, forma escara. Una vez diagnosticada, puede ser controlada con anfotericina $\mathrm{B}$ y desbridamiento conservador. En ausencia de este tipo de manejo, la micosis se disemina por vía hemática y progresa hasta dar formas graves. En la segunda forma ocurre separación de la fascia, fenómeno que es patognomónico de la fascitis necrosante $(17,20,23,24)$. Esta forma se caracteriza por su avance rápido, el cual va acompañado de ulceración, invasión vascular y trombosis de los vasos $(25,26)$, como fuera observado en el caso objeto de este informe. Inicialmente, pudimos observar una necrosis 'hepatizada', en la que la parte más superficial de la lesión secretaba material purulento gelatinoso o pastoso de color blanquecino, como fuera descrito anteriormente (5,21-24). En el caso de quemaduras, la laceración cuyo espesor es sólo parcial al inicio, progresa hasta que toda presenta el mismo aspecto, cuando en este momento aparece una escara central oscura, rodeada de un margen violáceo $(1,2,6,27)$, Este evento fue similar al observado en ciertas lesiones de nuestro paciente.

Una revisión de la literatura señala 37 informes sobre mucormicosis (zigomicosis) causada por A. elegans (cuadro 1) (11,25-50). El informe actual, el segundo en Latinoamérica, eleva a 38 el número de pacientes. De ellos, la gran mayoría $(86,5 \%)$ fueron hombres con edades variables entre 6 y 70 años, sin una distribución con diferencias acentuadas por grupo etario; su distribución geográfica señaló 17 casos en Estados Unidos, 12 en India, 4 en Australia, 2 en Méjico y 1 caso en cada uno de estos países: Antillas, Venezuela y Colombia (cuadro 1). En 27 pacientes (71\%), la infección se atribuyó a trauma, como el caso que se presenta; la ruta respiratoria se mencionó en 2 casos, mientras que en 5 no fue definida. A diferencia de otros agentes etiológicos, $A$. elegans se presenta casi siempre en pacientes inmunocompetentes, ya que $74 \%$ de los pacientes no presentó ninguna enfermedad de base en el momento del diagnóstico; para el resto se menciona: diabetes mellitus, alcoholismo y tratamiento con esteroides en pacientes con dermatitis crónica y sinusitis crónica. La mitad de los pacientes presentaba enfermedad diseminada a más de un órgano o sitio anatómico y si se toma como ejemplo el caso que se informa, $A$. elegans presenta tendencia importante a la diseminación (26,31-33,39,41,43).

A este respecto, se anota invasión rápida y amplia capacidad para invadir todo tipo de tejido $(11,26,28,31,41,48)$ y es frecuente el compromiso renal que se observa en 9 casos. La induración del tejido blando ocasionada por el hongo es uno de los hallazgos clínicos más constantes; ésta progresa alrededor de los bordes del sitio afectado, el cual toma el aspecto de una escara, lo que resulta en necrosis de tejido subcutáneo y músculo; ocasionalmente puede notarse crepitación $(23,31,33,39)$. Adam observó cómo el hongo se extendía por la fascia pero sin entrar al músculo (22). Vainrub y colaboradores reportaron cómo el tejido de granulación aparecía pocas semanas después, lo que permitía hacer injertos (10). En nuestro caso nunca existió mionecrosis pero tampoco se formó tejido de granulación. En forma similar a lo descrito en la literatura, observamos el crecimiento algodonoso del moho en la herida y en los bordes de la misma, poco sangrado cuando el tejido era desbridado en cirugía y apariencia necrótica de los bordes, como se aprecia en las figuras $(23,25,40,46)$.

En cuanto al agente etiológico, $A$. elegans es un moho perteneciente a la clase de los zigomicetes, orden Mucorales, familia Mucoraceae y género Apophysomyces; como tal, presenta hifas anchas (4-12 micras de diámetro), hialinas, ramificadas en ángulo recto, casi siempre aseptadas y de crecimiento rápido. Por consiguiente, sus colonias son algodonosas, altas y cubren rápidamente (25 días) la superficie del medio de cultivo. Característicamente, presenta apófisis en forma de embudo o campana y esporangias piriformes, si bien la esporulación típica no es fácil de obtener 
Cuadro 1. Mucormicosis (zigomicosis) por Apophysomyces elegans: resumen de los casos publicados.

\begin{tabular}{|c|c|c|c|c|c|c|c|c|c|c|}
\hline \multirow[t]{2}{*}{ Caso } & \multicolumn{3}{|c|}{$\begin{array}{c}\text { Datos demográficos } \\
\text { de base }\end{array}$} & \multirow[t]{2}{*}{$\begin{array}{l}\text { Enfermedad } \\
\text { lesión }\end{array}$} & \multirow[t]{2}{*}{$\begin{array}{l}\text { Localización } \\
\text { clínica }\end{array}$} & \multirow[t]{2}{*}{ Forma } & \multicolumn{2}{|c|}{ Terapia } & \multirow{2}{*}{$\begin{array}{l}\text { Super } \\
\text { viven- } \\
\text { cia }\end{array}$} & \multirow[t]{2}{*}{ Ref. } \\
\hline & $\begin{array}{l}\text { Edad } \\
\text { (años) }\end{array}$ & Sexo & País & & & & Anfo B & Cirugía & & \\
\hline 1 & 49 & $\mathrm{~F}$ & EE.UU. & $\begin{array}{l}\text { DM, Enf. } \\
\text { vascular }\end{array}$ & Pierna & C & $x$ & $x$ & Sí & 25 \\
\hline 2 & 56 & M & EE.UU. & No & $\begin{array}{l}\text { Riñón, vejiga, } \\
\text { flanco, tibia }\end{array}$ & $\mathrm{D}$ & $x$ & $\mathrm{x}$ & Sí & 26 \\
\hline 3 & 38 & M & EE.UU. & No & Muslo, glúteos & C & & $\mathrm{x}$ & Sí & 28 \\
\hline 4 & 45 & $\mathrm{M}$ & Australia & No & Rodilla & C & $x$ & & Sí & 27 \\
\hline 5 & 38 & $M$ & EE.UU. & No & Brazo, pierna & C & $x$ & $\mathrm{X}$ & No & 29 \\
\hline 6 & 59 & $\mathrm{M}$ & EE.UU. & No & Hombro & C & $x$ & $\mathrm{x}$ & Sí & 30 \\
\hline 7 & 27 & $\mathrm{M}$ & India & No & $\begin{array}{l}\text { Pared abdominal, } \\
\text { muslo, testículo, } \\
\text { pene }\end{array}$ & $\mathrm{D}$ & $x$ & $x$ & No & 31 \\
\hline 8 & 31 & M & EE.UU. & No & $\begin{array}{l}\text { Espalda, aorta, } \\
\text { riñón, pulmón, } \\
\text { intestino grueso } \\
\text { columna, lepto- } \\
\text { menínges }\end{array}$ & D & $x$ & & No & 32 \\
\hline 9 & 29 & $\mathrm{M}$ & EE.UU. & No & $\begin{array}{l}\text { Riñón, flancos } \\
\text { derecho e izquierdo }\end{array}$ & $\mathrm{D}$ & $x$ & $\mathrm{X}$ & Sí & 33 \\
\hline 10 & 70 & M & EE.UU. & No & $\begin{array}{l}\text { Piel de tórax, } \\
\text { esternón }\end{array}$ & C & $x$ & $\mathrm{X}$ & Sí & 34 \\
\hline 11 & 69 & M & Aruba & No & $\begin{array}{l}\text { Brazo, hombro, } \\
\text { húmero }\end{array}$ & C & $x$ & $x$ & Sí & 35 \\
\hline 12 & 19 & $\mathrm{M}$ & México & No & Rino-orbital & RO & $x$ & $\mathrm{X}$ & Sí & 11 \\
\hline 13 & 50 & $\mathrm{M}$ & EE.UU. & DM, & $\begin{array}{l}\text { Antebrazo } \\
\text { trasplante renal }\end{array}$ & C & $x$ & $\mathrm{X}$ & Sí & 36 \\
\hline 14 & 30 & $\mathrm{~F}$ & India & No & Brazo & C & & $\mathrm{x}$ & Sí & 37 \\
\hline 15 & 52 & $\mathrm{M}$ & India & $\begin{array}{l}\text { Mielo- } \\
\text { fibrosis, } \\
\text { hiper- } \\
\text { glucemia }\end{array}$ & Rino-orbital & RO & $x$ & $x$ & No & 38 \\
\hline 16 & 32 & $\mathrm{~F}$ & India & No & $\begin{array}{l}\text { Pared abdominal, } \\
\text { peritoneo, tórax, } \\
\text { fascitis necrosante }\end{array}$ & $\mathrm{D}$ & $x$ & $\mathrm{x}$ & Sí & 39 \\
\hline 17 & 34 & $\mathrm{M}$ & Venezuela & No & Muslo & C & $x$ & $\mathrm{X}$ & Sí & 40 \\
\hline 18 & 6 & M & EE.UU. & No & $\begin{array}{l}\text { Brazo, flancos, } \\
\text { riñón, espacio } \\
\text { peritoneal, bazo, } \\
\text { diafragma, colon, } \\
\text { tejido periaórtico. }\end{array}$ & $\mathrm{D}$ & $x$ & $x$ & Sí & 41 \\
\hline 19 & 54 & M & EE.UU. & $\begin{array}{l}\text { Sinusitis } \\
\text { crónica }\end{array}$ & Senos paranasales & RO & $x$ & $x$ & $x$ & 42 \\
\hline 20 & 6 & $\mathrm{M}$ & México & No & $\begin{array}{l}\text { Flanco, riñón, } \\
\text { espalda, páncreas, } \\
\text { mesenterio }\end{array}$ & $\mathrm{D}$ & $x$ & $x$ & No & 43 \\
\hline 21 & 55 & M & EE.UU. & $\begin{array}{l}\text { Alcoho- } \\
\text { lismo }\end{array}$ & Rodilla & $\mathrm{C}$ & $x$ & $x$ & Sí & 43 \\
\hline
\end{tabular}




\begin{tabular}{|c|c|c|c|c|c|c|c|c|c|c|}
\hline \multirow[t]{2}{*}{ Caso } & \multicolumn{3}{|c|}{$\begin{array}{c}\text { Datos demográficos } \\
\text { de base }\end{array}$} & \multirow[t]{2}{*}{$\begin{array}{l}\text { Enfermedad } \\
\text { lesión }\end{array}$} & \multirow[t]{2}{*}{$\begin{array}{l}\text { Localización } \\
\text { clínica }\end{array}$} & \multirow[t]{2}{*}{ Forma } & \multicolumn{2}{|c|}{ Terapia } & \multirow{2}{*}{$\begin{array}{l}\text { Super } \\
\text { viven- } \\
\text { cia }\end{array}$} & \multirow[t]{2}{*}{ Ref. } \\
\hline & $\begin{array}{l}\text { Edad } \\
\text { (años) }\end{array}$ & Sexo & País & & & & Anfo B & Cirugía & & \\
\hline 22 & 59 & M & Australia & No & Rinoorbitario & RO & $x$ & $x$ & Sí & 44 \\
\hline 23 & 24 & M & EE.UU. & No & Rinoorbitario & RO & $x$ & $\mathrm{x}$ & Sí & 45 \\
\hline 24 & 46 & M & Australia & DM & $\begin{array}{l}\text { Rodilla, espalda, } \\
\text { cresta ilíaca }\end{array}$ & $\mathrm{D}$ & $x$ & $x$ & No & 46 \\
\hline 25 & 22 & M & Australia & No & Brazo & C & $x$ & $\mathrm{x}$ & Sí & 46 \\
\hline 26 & 65 & M & EE.UU. & DM & Espalda & C & $x$ & $x$ & Sí & 47 \\
\hline 27 & 68 & M & EE.UU. & $\begin{array}{l}\text { DM } \\
\text { Dermatitis } \\
\text { crónica }\end{array}$ & Flanco izquierdo & C & $\mathrm{x}$ & $x$ & Sí & 48 \\
\hline 28 & 29 & M & EE.UU. & No & Muslo & $\mathrm{C}$ & $x$ & $\mathrm{x}$ & Sí & 23 \\
\hline 29 & 65 & M & India & No & Región lumbar & C & $\mathrm{X}$ & $x$ & No & 49 \\
\hline 30 & 20 & $\mathrm{~F}$ & India & No & $\begin{array}{l}\text { Septo nasal } \\
\text { y tejido retroorbitario }\end{array}$ & RO & $x$ & $x$ & Sí & 49 \\
\hline 31 & 35 & M & India & $\begin{array}{l}\text { Alcoho- } \\
\text { lismo }\end{array}$ & $\begin{array}{l}\text { Hidronefrosis bilateral } \\
\text { y pionefrosis derecha }\end{array}$ & $\begin{array}{ll}\text { al } \\
\text { a }\end{array}$ & $\mathrm{X}$ & $X$ & Sí & 49 \\
\hline 32 & 10 & M & India & No & $\begin{array}{l}\text { Pielonefritis y } \\
\text { abscesos en } \\
\text { ambos riñones }\end{array}$ & $\mathrm{D}$ & $x$ & $x$ & Sí & 49 \\
\hline 33 & 45 & M & India & No & $\begin{array}{l}\text { Bazo, colapso y } \\
\text { derrame pleural; } \\
\text { infarto en riñón. } \\
\text { celulitis flanco } \\
\text { izquierdo }\end{array}$ & D & $x$ & $x$ & No & 49 \\
\hline 34 & 25 & $\mathrm{~F}$ & India & $\mathrm{DM}$ & Glúteos & $\mathrm{C}$ & & $x$ & Sí & 49 \\
\hline 35 & 28 & M & India & No & Glúteos & $\mathrm{C}$ & & $x$ & Sí & 49 \\
\hline 36 & 70 & M & India & No & $\begin{array}{l}\text { Riñòn pelvis; } \\
\text { derrame pleural }\end{array}$ & $\mathrm{D}$ & & $x$ & No & 49 \\
\hline 37 & 31 & $\mathrm{~F}$ & & $\begin{array}{l}\text { No } \\
\text { (emba- } \\
\text { razo) }\end{array}$ & $\begin{array}{l}\text { Cabeza, } \\
\text { brazo }\end{array}$ & C & $x$ & $x$ & No & 50 \\
\hline 38 & 7 & M & Colombia & No & $\begin{array}{l}\text { Tórax, miembros } \\
\text { inferiores, pelvis, } \\
\text { testítulo, pene, } \\
\text { peritoneo, glúteos, } \\
\text { otros }\end{array}$ & $\mathrm{D}$ & $x$ & $x$ & $x$ & $\begin{array}{l}\text { Este } \\
\text { informe }\end{array}$ \\
\hline
\end{tabular}

DM: diabetes mellitus

Formas clínicas: C: cútanea; RO: rinocerebral: D: diseminada 
$(1,2,51)$. Crece bajo un amplio rango de temperaturas $\left(25^{\circ} \mathrm{C}\right.$ a $\left.55^{\circ} \mathrm{C}\right)$; las óptimas son las comprendidas entre $28^{\circ} \mathrm{C}$ y $30^{\circ} \mathrm{C}(1,2)$. A. elegans fue descrito por Misra y colaboradores en 1979 en India $(2,14)$ y aislado por primera vez en Estados Unidos a partir de lavado bronquial en 1982 (51); los primeros dos casos fueron informados por Winn y colaboradores en un congreso y registrados por Lawrence (26). Existen dos informes más referidos por Chakrabarti (49) que no se pudieron revisar e incluir en el cuadro 1 por falta de datos. A. elegans se encuentra distribuido en el suelo como contaminante ambiental, especialmente en áreas rurales de regiones tropicales y subtropicales $(1,2,6,14)$.

En el tejido, todos los mucorales presentan el mismo aspecto, hifas anchas de forma irregular con ramificaciones en ángulo recto. Se colorean con hematoxilina y eosina, así como con PAS o Gomori-Grocott. Generalmente, las hifas se observan en medio de un infiltrado neutrofílico de tipo agudo y con frecuencia se las ve invadiendo los vasos sanguíneos de la grasa subcutánea y de las fibras musculoesqueléticas con oclusión de los vasos, lo que causa trombosis, infartos, isquemia, necrosis y hemorragia $(1,2,18,25,26,43)$. La angio-invasión con trombosis y la necrosis de coagulación de los tejidos vecinos son hallazgos característicos (1,2,18,25-36,43), como se apreció en nuestro paciente.

Si bien la observación de las hifas ya descritas en especímenes clínicos, tanto en exámenes en fresco como en tejidos, es condición indispensable para establecer el diagnóstico, sólo el cultivo permite identificar la especie del hongo involucrado $(1,2,51)$. Además, el cultivo es la única vía definitiva para diferenciar entre mucormicosis y otras micosis como aspergilosis ya que en tejidos pueden confundirse las hifas si existen septos en el zigomiceto $(1,2,6,14)$. En forma similar, los bacilos Gram negativos, especialmente Pseudomonas, pueden causar lesiones vasculíticas de piel o vísceras que imitan las lesiones producidas por los Mucorales $(5,24,26)$.

En referencia al tratamiento y a pesar de que la terapia no está estandarizada $(1,2,6,15,17,19)$, todos los textos y artículos ofrecen un concepto único de manejo, haciendo énfasis en el diagnóstico precoz $(1,2,6,14,33,40)$. La sospecha clínica acompañada de la cirugía constituyen la mejor maniobra diagnóstica (13) y se aconseja hacer amplios y repetitivos desbridamientos $(8,10,12,17)$. Gordon y colaboradores consideran desbridar 2,5 cm más allá del área clínicamente comprometida, muchas veces, inicialmente cada 24 horas (7). En nuestro paciente se hallaron placas con actividad de $2 \mathrm{~cm}$ a $3 \mathrm{~cm}$ en los márgenes previamente resecados. En fascitis necrosante, el desbridamiento se considera adecuado cuando el dedo no diseca, lo cual facilita la separación de la grasa subcutánea de la fascia. El objetivo de la cirugía es la remoción de todo el tejido desvitalizado $(7,12)$.

La terapia antimicótica consiste en anfotericina $B$ (deoxicolato) o liposomal. La primera se da a dosis de 1-1,5 mg/kg por día para cualquier forma de mucormicosis (2); cuando se estabiliza el proceso, se disminuye la dosis a 0,8-1 mg/kg por día, con aplicación en días alternos $(1,2,15,17)$. La forma liposomal permite usar dosis mayores hasta de $10 \mathrm{mg} / \mathrm{kg}$ por día, con disminución de los efectos nefrotóxicos de la medicación clásica $(1,2,15)$. Sin embargo, por sí misma la droga suele ser insuficiente para controlar la infección por Mucorales $(2,34,44)$. El empleo de derivados azólicos ha sido limitado, especialmente en lo que a itraconazol y fluconazol se refiere ya que su efecto benéfico es mínimo (15). Sólo recientemente se ha tenido experiencia favorable con el nuevo derivado triazólico, posaconazol, si bien la experiencia es aún limitada (19). La literatura menciona también el uso de oxígeno hiperbárico, que puede ser coadyuvante; no obstante, no existen datos de medicina basada en la evidencia sobre su utilidad en mucormicosis $(1,2,12,45)$.

De los 38 pacientes informados (cuadro 1), todos menos uno $(97,3 \%)$ fueron sometidos a desbridamiento quirúrgico y 32 (84\%) fueron tratados con anfotericina B; los derivados azólicos y el oxígeno hiperbárico fueron de uso infrecuente.

Si se logra frenar la invasión por Mucorales, la cirugía reconstructiva juega un papel y es inevitable en la mayoría de casos. Requiere manejo interdisciplinario $(2,12)$. Generalmente, se emplean injertos de espesor parcial o colgajos 
una vez granulado el lecho cruento $(12,16)$. Se ha empleado vacuum-assisted clouse (VAC) para disminuir defectos cutáneos e injertar o hacer colgajos rotacionales o libres para cubrir hueso, cartílago, tendón o estructuras neurovasculares expuestas. Los procedimientos quirúrgicos se deben practicar rápidamente con el fin de preservar la función tisular y prevenir posibles problemas en las heridas $(12,16)$.

La duración del tratamiento depende de la respuesta del paciente, de la enfermedad de base y de la naturaleza y grado de toxicidad de la anfotericina $B(1,2,6,14,15)$. En la mucormicosis (zigomicosis) es difícil determinar correctamente la efectividad de cualquier abordaje terapéutico. La enfermedad es rara y no permite realizar estudios comparativos controlados, por lo que muchos de los casos aparecen en la literatura solamente si la terapia resultó efectiva $(1,2)$. En nuestro paciente y ante la sospecha inicial de un proceso de origen bacteriano, se emplearon antibióticos diversos antes del establecimiento del diagnóstico definitivo de mucormicosis. El hemoleucograma y los altos niveles de los reactantes de fase aguda demuestran infección pero no revelan una diferencia característica que permita sospechar infección por hongos $(1,2)$.

La extensión de las lesiones de nuestro paciente y su gran trastorno funcional llevaron a la suspensión de toda acción terapéutica, ofreciendo solo tratamiento paliativo; la muerte se presentó al término de la octava semana del accidente traumático que permitió la inoculación de $A$. elegans. Sólo se encontró un informe en el cual, a pesar del marcado déficit funcional, se continuó con el tratamiento hasta la muerte del paciente (41). A pesar de los tratamientos combinados, la mortalidad ocurre típicamente como resultado de extensión de la infección dentro de regiones vitales, donde la resección completa se hace imposible aun con dosis altas de anfotericina $B$ (2). En nuestro caso fue la severidad de la extensión y el compromiso de vida a futuro, los hechos que imposibilitaron la recuperación del paciente.

Es conocido que las infecciones necrosantes no controladas progresan y llevan al paciente a falla orgánica multisistémica y a su muerte. La mortalidad global es de $38 \%$, pero depende de la edad y es tan alta como $67 \%$ en pacientes mayores de 50 años; influye también el grado de compromiso del tronco, la presencia de diabetes mellitus, así como la demora para establecer el diagnóstico e iniciar el tratamiento (2). Según Parfrey, la supervivencia es de $73 \%$ (18); según Adam es de $67 \%$ (22), y para la forma cutánea de $10 \%$. La supervivencia en la serie de 38 pacientes aquí revisados fue de $71 \%$ (cuadro 1); no obstante, si se considera que la mayoría de los pacientes son inmunocompetentes, una mortalidad de $29 \%$ es demasiado elevada.

En cuanto al pronóstico, la forma cutánea tiene mayores probabilidades de curación que otras formas de mucormicosis (2), pero la morbilidad a largo plazo aún es considerable. Por otra parte, no existen factores que permitan predecir el resultado final del paciente. Existe mucha variabilidad relacionada con el momento de iniciar el tratamiento y con la severidad de la infección (2). En mucormicosis primaria asentada en heridas traumáticas, el resultado depende de la localización, la extensión de las lesiones, la rapidez en el diagnóstico y la enfermedad de base $(1,2,6,26,42)$.

Se asocian con mal pronóstico el compromiso de cabeza o tronco $(1,38,50)$ a diferencia de las lesiones en las extremidades, las cuales son de baja mortalidad individual y pueden resolverse con la amputación $(1,2,8,27,30,35)$. Se ha sugerido que el estrés por trauma o posquirúrgico sería un factor predisponente a la infección ya que al deteriorarse el paciente, se presenta acidosis, condición que favorece la proliferación de los mucorales $(9,10,22)$. En 1992, Cocanour y colaboradores (8) revisaron por un periodo de 9 años, 14.262 pacientes con trauma (heridas contaminadas o abiertas) a su ingreso al hospital y encontraron solamente 11 pacientes con diagnóstico comprobado de mucormicosis, 9 con trauma contuso y 2 con quemaduras mayores del 50\%; 7 habían presentado choque hipovolémico o acidosis en la hospítalización. Todos recibieron antibiótico de amplio espectro antes de desarrollar la mucormicosis (8).

Existen fallas médico-legales en lo que a diagnósticos incorrectos o tardíos se refiere, los que son comunes en todos los casos revisados 
en la literatura, incluido el nuestro, lo cual indica que los médicos normalmente no asocian los hongos con infecciones necrosantes $(1,2,34,35)$. Esto permite el avance de la infección, la necesidad de extensos desbridamientos, las cirugías mutilantes y las dosis altas de anfotericina B (43). También se falla por esperar el resultado de los cultivos, olvidando que los exámenes directos pueden proporcionar un diagnóstico en pocas horas y la biopsia en un par de días.

Una vez controlada la infección en los pacientes con fascitis necrosante, los colgajos de piel no se pierden porque el tejido de celulitis se resuelve rápidamente con tratamiento apropiado, dejando la piel viable. Si se falla en la reexploración de la herida, puede ocurrir el progreso de la infección en tejidos previamente sanos. Esto es especialmente peligroso en lesiones de tronco en pacientes que permanecen sépticos clínicamente, que tienen nutrición enteral y que están recibiendo antibióticos de acuerdo con el germen asociado y su sensibilidad $(12,16)$.

Obviamente, nada es más importante que tener en mente la mucormicosis en pacientes en riesgo para intentar establecer tempranamente el diagnóstico. El trabajo a futuro consiste en tratar de restaurar e incrementar la resistencia del hospedero, desarrollar métodos que permitan evaluar la anormalidad de los mecanismos de defensa y determinar la actividad de las nuevas drogas antifúngicas.

\section{Agradecimientos}

La amable cooperación prestada por los médicos pediatras, el personal de enfermería del Servicio de Pediatría del Hospital Pablo Tobón Uribe, así como de la sicóloga Sandra Alzate, es reconocida con gratitud. Pero ante todo, recordamos al paciente por las enseñanzas de vida que nos dejara y por su capacidad de renuncia.

\section{Referencias}

1. Ribes JA, Vanover-Sams CL, Baker DJ. Zygomycetes in human disease. Clin Microbiol Rev 2000;13:236-301.

2. Sugar AM. Agents of mucormycosis and related species. En: Mandell GL, Bennett JE, Dolin R, editors. Principles and practice of infectious diseases. Fifth edition. Philadelphia: Churchill Livingstone; 2000. p.268595.
3. Yeung CK, Cheng VC, Lie AK, Yuen KY. Invasive disease due to Mucorales: a case report and review of the literature. Hong Kong Med J 2001;7:180-8.

4. Chakrabarti A, Das A, Sharma A, Panda N, Das S, Gupta KL, Sakhuja V. Ten years' experience in Zygomycosis at a tertiary care centre in India. J Infect 2001;42:261-6.

5. Patiño JF. Infecciones necrotizantes de la piel y de los tejidos blandos. Rev Col Cirugía 1987;11:11-8.

6. Ibrahim AS, Edwards JE, Filler SG. Zygomycosis. En: Dismukes WE, Pappas PG, Sobel J, editors. Clinical mycology. New York, NY: Oxford University Press; 2003. p.241-51.

7. Gordon G, Indeck M, Bross J, Kapoor DA, Brotman S. Injury from silage wagon accident complicated by mucormycosis. J Trauma 1988;28:866-7.

8. Cocanour CS, Miller-Crotchett P, Reed L, Johnson $\mathbf{P}$, Fischer R. Mucormycosis in trauma patients. J Trauma 1992;32:12-5.

9. Johnson PC, Satterwhite TK, Monheit JE, Parks D. Primary cutaneous mucormycosis in trauma patients. J Trauma 1987;27:437-41.

10. Vainrub B, Macareno A, Mandel S, Musher D. Wound zycomycosis (mucormycosis) in otherwise healthy adults. Am J Med 1988;84:546-8.

11. Radner AB, Witt MD, Edwards JE. Acute invasive rhinocerebral zygomycosis in an otherwise healthy patient: case report and review. Clin Infect Dis 1995; 20:163-6.

12. Loose JE, Selber J, Vega S, Hall C, Scott G, Serletti JM. Primary cutaneous mucormycosis: guide to surgical management. Ann Plast Surg 2002;49:385-90.

13. Parfey NA. Improved diagnosis and prognosis of mucormycosis. Medicine 1986;65:113-23.

14. Prabhu RM, Pate IR. Mucormycosis and entomphthoromycosis: a review of the clinical manifestations, diagnosis, and treatment. Clin Microbiol Infect 2004;10 (Suppl.1):31-47.

15. Herbrecht R, Letscher-Bru V, Bowden RA, Kusne S, Anaissie EJ, Graybill JR et al. Treatment of 21 cases of invasive mucormycosis with amphotericin B colloidal dispersion. Eur J Clin Microbiol Infect Dis 2001; 20:460-6.

16. Sutherland ME, Meyer AA. Necrotizing soft-tissue infections. Surg Clin North Am 1994;74:591-607.

17. Jimenez C, Lumbreras C, Aguado JM, Loinaz C, Paseiro G, Andres A et al. Successful treatment of mucor infection after liver or pancreas-kidney transplantation. Transplantation 2002;73:476-80.

18. Pfaller MA, Messer SA, Hollis RJ, Jones RN. Antifungal activities of posaconazole, ravuconazole, and voriconazole compared to those of itraconazole and 
amphotericin B against 239 clinical isolates of Aspergillus spp. and other filamentous fungi: report from SENTRY Antimicrobial Surveillance Program, 2000. Antimicrob Agents Chemother 2002;46:1032-7.

19. Tobón AM, Arango M, Fernández D, Restrepo A. Mucormycosis (Zygomycosis) in a heart-kidney trasplant recipient: recovery after posaconazole therapy. Clin Infect Dis 2003;36:1488-91.

20. Trent JT, Kirsner RS. Necrotizing fasciitis. Wounds 2002;14:284-92.

21. Gartenberg G, Bottone EJ, Keusch GT. Hospitalacquired mucormycosis of skin and subcutaneous tissue: epidemiology, mycology and treatment. N Eng J Med 1978;299:1115-8.

22. Adam RD, Hunter G, Di Tomasso J, Comerci G. Mucormycosis: emerging prominence of cutaneous infections. Clin Infect Dis 1994;19:67-76.

23. Dowdy LM, DO, Castro JG, Duchesne C, Cleary T. Necrotizing fasciitis from Apophysomyces elegans Infection following trauma. Infect Med 2002;19:2869.

24. Kovacs LH, Kloeppel, Papadopulos NA, Reeker W, Niemer E. Necrotizing fasciitis. Ann Plast Surg 2001; 47:680-1.

25. Wieden MA, Steinbronn KK, Padhye AA, Ajello L, Chandler FW. Zygomycosis caused by Apophysomyces elegans. J Clin Microbiol 1985;22:522-6.

26. Lawrence RM, Snodgrass WT, Reichel GW, Padhye AA, Ajello L, Chandler FW. Systemic zygomycosis caused by Apophysomyces elegans. J Med Vet Mycol 1986;24:57-65.

27. Cooter RD, Lim IS, Ellis DH, Leitch IOW. Burn wound zygomycosis caused by Apophysomyces elegans. J Clin Microbiol 1990;28:2151-3.

28. Newton WD, Cramer FS, Norwood SH. Necrotizing fascitis from invasive Phycomycetes. Crit Care Med 1987:15:331-2

29. Huffnagle KE, Southern PM, Byrd LT, Gander RM. Apophysomyces elegans as an agent of zygomycosis in a patient following trauma. J Med Vet Mycol 1992;30: 83-6.

30. Weinberg WG, Wade BH, Cierny G III, Stacy D, Rinaldy MG. Invasive infection due to Apophysomyces elegans in immunocompetent hosts. Clin Infect Dis 1993;17: 881-4.

31. Lakshmi V, Rani TS, Sharma S, Mohan VS, Sundaram C, Rao RR, Satyanarayana G. Zygomycotic necrotizing fasciitis caused by Apophysomyces elegans. J Clin Microbiol 1993;31:1368-9.

32. McGinnis MR, Midez J, Pasarell L, Hake A. Necrotizing fasciitis caused by Apophysomyces elegans. J Mycol Med 1993;3:175-9.
33. Okhuysen PC, Rex JH, Kapusta M, Fife C. Successful treatment of extensive posttraumatic soft-tissue and renal infections due to Apophysomyces elegans. Clin Infect Dis 1994;19:329-31.

34. Eaton ME, Padhye AA, Schwartz DA, Steinberg JP. Osteomyelitis of the sternum caused by Apophysomyces elegans. J Clin Microbiol 1994;32:2827-8.

35. Meis JFG, Kullberg B, Pruszczynski M, Veth RPH. Severe osteomyelitis due to the zygomycete Apophysomyces elegans. J Clin Microbiol 1994;32:3078-81.

36. Naguib MT, Huycke MM, Pederson JA, Pennington LR, Burton ME, Greenfield RA. Apophysomyces elegans infection in a renal transplant recipient. Am J Kidney Dis 1995;26:381-4.

37. Chakrabarti A, Kumer P, Padhye AA, Chatha L, Singh SK, Das A et al. Primary cutaneous zygomycosis due to Saksenaea vasiformis and Apophysomyces elegans. Clin Infect Dis 1997;24;580-3.

38. Chakrabarti A, Panda N, Varma SC, Singh K, Das A, Sharma SC et al. Craniofacial zygomycosis caused by Apophysomyces elegans. Mycoses 1997;40:419-21.

39. Mathews MS, Raman A, Nair A. Nosocomial zygomycotic post-surgical necrotizing fasciitis in a healthy adult caused by Apophysomyces elegans in South India. J Med Vet Mycol 1997;35:61-3.

40. Cáceres AM, Sardiñas C, Marcano C, Guevara R, Barros J, Bianchi G et al. Apophysomyces elegans limb infection with a favourable outcome: case report and review. Clin Infect Dis 1997;25:31-2.

41. Burell SR, Ostlie DJ, Saubolle M, Dimler M, Barbour SD. Apophysomyces elegans infection associated with cactus spine injury in an immunocompetent pediatric patient. Pediatr Infect Dis J 1998;17:663-4.

42. Brown SR, Shah IA, Grinstead M. Rhinocerebral mucormycosis caused by Apophysomyces elegans. Am J Rhinol 1998;12:289-292.

43. Kimura M, Smith MB, McGinnis MR. Zygomycosis due to Apophysomyces elegans. Arch Pathol Lab Med 1999;123:386-90.

44. Fairley C, Sullivan TJ, Bartley P, Allworth T, Lewandowski R. Survival after rhino-orbital-cerebral mucormycosis in an immunocompetent patient Opthalmology 2000;107:555-8.

45. Garcia-Covarrubias L, Barlett R, Barrat DM, Wassermann RJ. Rhino-orbitocerebral mucormycosis attributable to Apophysomyces elegans in an immunocompetent individual: case report and review of the literature. J Trauma Inj Infect Crit Care 2001;50:353-7.

46. Page R, Gardam DJ, Heath C. Severe cutaneous mucormycosis (zygomycosis) due to Apophysomyces elegans. Aust J Surg 2001;71:184-6. 
47. Blair JE, Fredrikson LJ, Pockaj BA, Lucaire CS. Locally invasive cutaneous Apophysomyces elegans infection acquired from snapdragon patch test. Mayo Clin Proc 2002;77:717-20.

48. LeSueur BW, Warschaw K, Fredrikson L. Necrotizing cellulites caused by Apophysomyses elegans at a patch test site. Am J Contact Dermatitis 2002;13:140-2.

49. Chakrabarti A, Ghosh A, Prasad GS, David JK, Grupta S, Das A, et al. Apophysomyces elegans: an emerging zygomycete in India. J Clin Microbiol 2003;41: 783-8.

50. Carter JE, Ulusarac $\mathbf{O}$. Widespread cutaneous involvement by invasive Apophysomyses elegans in a gravid patient following trauma. Cutis 2003;72:221-4.

51. Ellis J, Ajello J. An inusual source of Apophysomyces elegans and a method for stimulating sporulation of Saksenaea vasiformis. Mycologia 1982;74:144-5. 